TEMAS DE ACTUALIDAD Rev Chil Salud Pública 2015; Vol 19 (1): 64-68

\section{EL ACTA COMO INSTRUMENTO DE GESTIÓN DE CAMBIO: UN ESTUDIO DE CASO}

\author{
Meeting Minutes as instruments for Change management: \\ A CASE STUDY
}

\section{INTRODUCCIÓN}

El 2013 comenzó una intervención para la Acreditación de Prestadores en el área de salud de la Corporación de Desarrollo Social de Providencia. Luego de un diagnóstico organizacional se decidió con el cliente, realizar una intervención integral que, manteniendo un foco en calidad, permitiera una re-dirección estratégica.

Se usó el enfoque de investigación-acción, junto con actividades estándares de gestión de cambio, y se complementó con el uso de un registro ${ }^{(i)}$ (el acta de reuniones).

La labor gerencial en organizaciones de salud, y otros sectores, se caracteriza por lo breve y variado de sus actividades, la fragmentación y discontinuidad del trabajo, y su preferencia por modalidades informales y orales de comunicación, ${ }^{1}$ luego coordinación y control se vuelven problemas críticos.

La forma habitual de coordinación entre unidades son los comités, los que en reuniones concuerdan programas, cronogramas, y asignan responsabilidades, con el eventual apoyo de software de seguimiento de mayor o menor complejidad y costo.

Un problema adicional en salud pública es que los comités pueden ser de naturaleza clínica, técnico-administrativa, docentes, de gestión clínica, o una fuerza de tarea para un proyecto. ¿Cómo comparar la efectividad de cada uno?

Este estudio de caso tiene una importancia práctica, al aportar a los directivos de organizaciones de salud pública un procedimiento sencillo y de bajo costo para gestionar los comités que crean para la coordinación y control de tareas.

Este trabajo trata de resolver el problema de tener una metodología que mejore la coordinación y control de los equipos de salud, mediante un instrumental de bajo costo, y que permita comparar la efectividad de equipos de naturaleza diferentes. Se plantearon las siguientes preguntas: ¿Se pueden usar las actas como instrumento de gestión?; y ¿Se puede evaluar de manera sencilla la efectividad de los diversos comités mediante sus actas?

Recibido 2 de agosto 2014; aceptado 10 de enero 2015

(i). La norma ISO 9001:2008 incluso establece en su ítem normativo 4.2.4 el "control de los registros”. 
El objetivo de la investigacion fue describir un método para usar las actas como instrumento de gestión, y describir un método de evaluación de la efectividad de los diversos comités mediante sus actas.

\section{MARCO TEÓRICO}

El sistema de salud puede ser descrito desde la teoría autopoiética, ${ }^{2}$ como formado por comunicación que produce comunicación. ${ }^{3}$ Actúa por medio de organizaciones de salud que se pueden describir como formadas por decisiones que producen decisiones. ${ }^{4}$

Las decisiones en las organizaciones dicen relación con los medios (recursos) y con los fines (objetivos), ${ }^{4}$ con la pertenencia (membresía), y con los procedimientos; ${ }^{5}$ estas decisiones son comunicación, es decir, algo que es dicho de una manera, y que es entendido por alguien. ${ }^{6}$

La producción de decisiones no se puede detener sin detener la autopoiesis, luego algunas decisiones son almacenadas en manuales, procedimientos, instrucciones y registros esperando ser usadas.

Las decisiones se producen en el marco de un conflicto que puede ser funcional si se centra en tareas y temas, o disfuncional si lo hace sobre personas y relaciones. ${ }^{?}$

En los cambios organizacionales los factores que impulsan el cambio entran en conflicto con los factores que lo obstaculizan. ${ }^{8} \mathrm{Un}$ cambio organizacional que afecte la forma de solucionar problemas de integración y adaptación de la organización, ${ }^{9}$ debe mantener la autopoiesis, manteniendo el sentido de urgencia. ${ }^{10}$

\section{DESCRIPCIÓN DEL CASO}

\section{Situación en febrero de $2013^{\text {(ii) }}$}

Providencia tenía una población estimada de 131.708 habitantes, $54 \%$ mujeres, $23 \%$ adultos mayores (media nacional 17\%), 33,6\%

(ii). Los siguientes datos se extrajeron del documento Ejes Estratégicos en Salud de la Dirección de Salud, Corporación de Desarrollo Social de Providencia, 2013. es económicamente activo, $56,8 \%$ de los hogares tiene computador. ${ }^{\text {(iii) }}$ Los principales problemas de salud son cardiovasculares, respiratorios, de salud mental, osteoarticulares, tumorales y de salud dental.

La Dirección de Salud de la Corporación de Desarrollo Social administra los centros. El 2013 existían dos CESFAM (Dr. Hernán Alessandri y El Aguilucho), con cinco sectores; un Centro de Salud Mental (PROVISAM), y un Centro Dental (Dr. Alfonso Leng), en donde funcionaba el Centro de Especialidades Odontológicas (CEO), todos certificados ISO 9001.

La infraestructura fue diseñada para una población de 23 mil personas y la inscrita y validada el 2012 era de $47.328^{\text {(iv) }}$ usuarios, de los cuales $28 \%$ eran adultos mayores.

El modelo de atención era el de consultorio general urbano, centrado en las consultas de morbilidad. La necesidad de acreditar los centros de salud requería re-diseñar el Sistema de Gestión de Calidad basado en la ISO 9001:2008.

El diagnóstico organizacional identificó un deterioro del liderazgo, daño al clima laboral, resistencia al cambio de una nueva ficha electrónica, nula adhesión al sistema ISO. En Focus Group de los equipos de trabajo en los centros, se reflejó descoordinación y centros operacionalmente aislados.

\section{Cambios hasta julio 2014 ${ }^{(v)}$}

En febrero del 2013 se definieron tres ejes estratégicos: la Red de Salud comunal (conjunto de unidades de salud que producen atenciones de salud); un Programa Escolar de Salud (PES) para la atención de estudiantes de los colegios municipales; y un Programa de Calidad Mayor (PCM) orientado al adulto mayor. Los tres ejes se integraron en el Plan de Salud 2014.

Se crearon unidades de salud de tamaño diverso, desde un CECOF hasta salas de

\footnotetext{
(iii). Censo 2002.

(iv). Población percapitada por Fonasa para el año 2012.

(v). La siguiente descripción corresponde a los principales cambios contenidos en los registros.
} 
procedimientos en centros comunitarios, con ficha electrónica en toda la red. El PES creó unidades en cada colegio, el PCM abrió unidades en los centros de Adulto Mayor Municipal; ambos desarrollan actividades de tratamiento, promoción y prevención.

Se reconvirtió el Centro Dental en un Centro de Salud, y se autonomizó el Centro de Especialidades Odontológicas; se crearon tres unidades comunales: un Centro de Esterilización, una Bodega de Farmacia y una Plataforma de Atención al Usuario.

Se implementó el modelo de Salud Familiar pasando de 5 a 7 sectores, se reforzó a los jefes de sector dándoles gestión de su equipo, se convirtió a los jefes de programa en asesores técnicos, se creó el cargo de jefe de servicios de apoyo, y se concursaron las tres direcciones de centro usando el método de assessment center. Se rediseñó la carrera funcionaria con los gremios.

La Dirección de Salud tiene el PES, PCM, un departamento de Gestión de la Información en Salud (controla indicadores y uso de ficha electrónica), un departamento de Promoción, Prevención y Participación en Salud, y un staff de Calidad a cargo del Sistema de Gestión de Calidad (integra ISO 9001:2008 y Acreditación de Prestadores de Atención Abierta).

Los cambios se coordinaron mediante comités estables:

1. Comité de Directores de Centro (CDC): Director de Salud, jefes de departamentos y programas, direcciones de centros.

2. Comité Comunal de Calidad (CC): staff de Calidad y encargados locales de calidad.

3. Comité de la Dirección (C de D): Director de Salud, jefaturas de la Dirección.

4. Comité de Especialidades (CE): Director de Salud, directivos del COSAM y del CEO.

5. El Comité Ampliado Comunal (CA): los señalados, más las jefaturas de Esterilización, Bodega de Farmacia y Plataforma de Atención.

Además, existen comités temporales por proyectos.
Respecto de los comités, se usó un diseño de acta que consta de cuatro partes:

Primera sección de identificación: nombre del comité que se reúne, fecha, hora de inicio y término, lugar, asistentes con su nombre y cargo.

Segunda sección de texto: incluye los principales tópicos tratados en la reunión, descritos en tercera persona, se deja constancia de la revisión de los acuerdos previos, si corresponde.

Tercera sección de acuerdos numerados: descritos en términos operativos, con responsables y si corresponde con fecha de ejecución.

Cuarta sección con la tabla de la siguiente reunión: fecha y hora, y dos puntos mínimos (1. la "revisión de los acuerdos" y 2. un “otros”), más puntos adicionales.

El acta se elabora dentro de las 24 a 48 horas post reunión, y se envía por mail a todos los asistentes, con copia a otros cuya actividad puede verse afectada por los acuerdos. Si existen observaciones se modifica.

En la mayoría de las reuniones se comienza revisando los acuerdos previos uno por uno, se etiqueta su situación como "cerrado" si se ha ejecutado, o "pendiente" si no se ha iniciado su ejecución o no se ha terminado. Los acuerdos pendientes pasan a la sección "acuerdos" de la nueva acta y se mantienen en el tiempo, revisándose en cada reunión hasta su cierre, que puede ser por cumplimiento o imposibilidad de cumplimiento.

\section{CARACTERIZACIÓN DE LA INVESTIGACIÓN}

Es un estudio de caso, en un marco epistemológico constructivista, descriptivo, cualicuantitativo y usa fuentes primarias.

\section{METODOLOGÍA}

\section{Muestra}

Se elaboraron 109 actas, las que corresponden a los comités de: Directores de centros; Jefes de departamentos y programas de la Dirección 
de Salud; Calidad Comunal; Ampliado; Especialidades; Staff de Calidad; Otros temporales: concurso de directores, reunión de alta dirección (ISO).

Los criterios de selección de la muestra de actas fueron: 1) Solo aquellas elaboradas por el investigador; 2) Reuniones de comités estables o formados por miembros estables (estabilidad implica un mínimo de seis meses de trabajo conjunto de la mayoría de los miembros).

Al aplicar los criterios de selección las actas analizables se redujeron a 79: Comité de Directores de Centro (40); Comité de Dirección de Salud (27); Comité de Calidad Comunal (7); Comité Ampliado (2); Comité de Especialidades (3).

\section{Técnica de investigación}

Las 79 actas fueron revisadas, en cada una se contaron los acuerdos tomados (tercera sección), el aspecto cualitativo de la investigación radicó en la codificación de la revisión de los acuerdos previos (segunda sección), ya sea como "cerrado" si es que el acuerdo se realizó, o "pendiente”, si no se hizo.

Luego se procedió dicotómicamente a dar el valor 1 al acuerdo "cerrado" y el valor 0 al "pendiente".

El número de acuerdos (producción), y los valores de acuerdos "cerrados" o "pendientes" (eficacia) se llevaron a una planilla Excel para obtener datos estadísticos simples, como promedios, modas y medianas.

Respecto de correlaciones se hicieron entre el "número de acuerdos" y "cumpli- miento de acuerdos" solo en las actas que consignan revisión de acuerdos, para el total y para los dos comités con mayor cantidad de actas (CDC, C de D).

\section{RESULTADOS}

En el total de la muestra el promedio de "acuerdos tomados" es de 9,52 (moda de 8 y mediana de 8,5 ), el "nivel de cumplimiento" promedio es de $51,59 \%$, con un promedio de “acuerdos cumplidos" de 4,81 acuerdos por reunión (moda de 1 y mediana de 4).

En los comités individuales el promedio de acuerdos va de 6 en CA a 11,47 en CDC, y el nivel de cumplimiento varía entre $30 \%$ en el CA a $69,57 \%$ en el CC.

La correlación entre "acuerdos tomados" y "nivel de cumplimiento" es de $-0,33$, para todas las actas que consignan revisión de acuerdos. En los dos comités con mayor cantidad de actas (CDC y C de D), la correlación también es negativa pero con distinta magnitud, para CDC es de - 0,57 , y para C de D es de $-0,04$.

\section{DISCUSIÓN}

Los comités son diversos, el CDC es de gestión clínica y administrativa, el CC es temático, el $\mathrm{C}$ de $\mathrm{D}$ es administrativo, el CE es de gestión clínica y administrativa de nivel secundario, y el CA de gestión administrativa de la red.

Tabla por Comité

\begin{tabular}{lcc}
\hline Comité & Promedio acuerdos por reunión (en \%) & Nivel cumplimiento por reunión (en \%) \\
\hline Total & 9,5 & 51,6 \\
Directores de centro (CDC) & 11,5 & 54,7 \\
Dirección de salud (C de D) & 6,6 & 40,4 \\
Calidad comunal (CC) & 9,7 & 69,6 \\
Ampliado (CA) & 13,5 & 30 \\
Especialidades (CE)* & 6 & NA \\
\hline
\end{tabular}

* Ha tenido tres reuniones y solo en una se hizo revisión de acuerdos. 
$\mathrm{Al}$ medir las variables "número de acuerdos" y "nivel de cumplimiento", los resultados totales permiten establecer una línea base (promedio total) de producción de acuerdos y su cumplimiento, contra la cual comparar cada comité.

El C de D resulta el menos productivo con 6,5 acuerdos por reunión, el menos eficaz es el CA con el $30 \%$ de cumplimiento, aunque es el que más produce acuerdos por reunión $(13,5)$ y el CC resulta el más eficaz con un $69,57 \%$ de cumplimiento.

La correlación de $-0,33$, en actas en que se revisaron acuerdos, indica que las variables se mueven en direcciones opuestas. Esto se puede explicar por la acumulación de acuerdos, al trasladar "pendientes" de una reunión a la siguiente. Esto se entiende en el contexto de los cambios descritos del área de salud, por la curva de experiencia y la caída de productividad asociada a toda gestión del cambio.

La diferencia en producción y eficacia permite al gestor focalizar esfuerzos de mejora, por ejemplo interviniendo en el C de D para aumentar la producción de decisiones mediante lluvia de ideas o Delphi, y/o mejorar el nivel de ejecución del CA mediante mentoring, coaching o trabajo en equipo.

Para efectos académicos el material (actas) podría ser usado para analizar hipótesis respecto de los factores que afectan la producción o el cumplimiento, como la composición de los comités (profesión, edad, asistencia), los temas tratados (diversidad), o el tiempo destinado, además se puede hacer análisis de discurso, como el de enmarcado (frame) para hacer un análisis de alineamiento.

En sintesis, el trabajo describe cómo usar las actas para gestionar: el proceso considera tres actividades: primero, la construcción de las actas; segundo, la verificación del cumplimiento de acuerdos, ambas hechas por un secretario ejecutivo, el rol del líder es avalar e incentivar -la rendición de cuentas ante pares ha demostrado ser una práctica útil ${ }^{11}$-; tercero, la revisión trimestral o semestral de una muestra de actas, por un profesional del área de control o calidad.
Se lleva la muestra a una planilla Excel dando valor 1 al acuerdo "cerrado" y valor 0 al "pendiente", estableciendo una línea estándar (promedios totales) contra la cual comparar equipos de trabajo diferentes. Los resultados permiten decidir intervenciones futuras.

\section{AGRADECIMIENTOS}

Agradezco al Sr. Marcelo Belmar, Director de Salud de la Corporación de Desarrollo Social de Providencia, a los jefes(as) de departamentos y programas, a directivos y jefaturas de centros y a todo el personal de la Red de Salud Municipal.

\section{REFERENCIAS}

1. Mintzberg H. Managing. Bogota: Editorial Norma; 2010.

2. Maturana H, Varela F. El árbol del conocimiento. $8^{\text {a }}$ ed. Santiago: Editorial Universitaria; $1992.171 \mathrm{pp}$.

3. Luhmann N. La sociedad de la sociedad. México: Herder, 2007.

4. Organización y decisión. Autopoiesis, acción y entendimiento comunicativo. España: Anthropos; 1997.

5. Rodríguez D, Arnold M. Sociedad y teoría de sistemas. $4^{\mathrm{a}}$ ed. Santiago: Editorial Universitaria; 2007.

6. Farías I, Ossandón J. Observando Sistemas. Santiago: RIL editores; 2006.

7. Robbins S, Coulter M. Administración. $5^{\mathrm{a}}$ ed. México: Prentice Hall; 1996.

8. Kast Fremont E, Rosenzweig JE. Administración en las organizaciones, enfoque de sistemas $y$ de contingencias. México: McGraw-Hill; 1988.

9. Acuña E. La transición de empresa pública a privada: cambio cultural. Chile, Universidad de Chile; 1995.

10. Kotter J. El sentido de la urgencia. Bogotá: Editorial Norma; 2009.

11. Chan kin W., Mauborgne R. La estrategia del océano azul. Bogotá: Editorial Norma; 2008. 